\title{
CESÀRO CONVERGENCE OF MARTINGALE DIFFERENCE SEQUENCES AND THE BANACH-SAKS AND SZLENK THEOREMS
}

\author{
FRANCISCO J. FRENICHE
}

(Communicated by William J. Davis)

\begin{abstract}
It is shown that, for $1 \leq p<+\infty$, any weakly null martingale difference sequence in $L^{p}[0,1]$ is Cesàro convergent to zero in the $L^{p}$ norm. This result combined with a theorem of Gaposhkin gives an easy proof of two theorems of Banach-Saks and Szlenk at once.
\end{abstract}

Introduction. We first recall the concept of martingale difference sequences. Let $\left(\Sigma_{n}\right)$ be a nondecreasing sequence of sub- $\sigma$-algebras of $\Sigma$, the algebra of Lebesgue measurable sets in $[0,1]$. If, for each $n, f_{n}$ is a $\Sigma_{n}$-measurable function whose conditional expectation with respect to $\Sigma_{n-1}$ satisfies $E\left(f_{n}, \Sigma_{n-1}\right)=0$, we then say that the sequence $\left(f_{n}\right)$ is a martingale difference sequence (m.d.s.) adapted to $\left(\Sigma_{n}\right)$. If every $f_{n}$ is in $L^{p}=L^{p}[0,1]$, we then say that $\left(f_{n}\right)$ is an m.d.s. in $L^{p}$, where $1 \leq p<+\infty$.

Now we can state Gaposhkin's theorem (see $[\mathbf{G}]$ ):

THEOREM A. Let $\left(g_{n}\right)$ be a weakly null sequence in $L^{p}$. Then there exists an m.d.s. $\left(f_{n}\right)$ in $L^{p}$ and a subsequence $\left(h_{n}\right)$ of $\left(g_{n}\right)$ such that the series $\Sigma_{n}\left\|h_{n}-f_{n}\right\|_{p}$ converges.

PROOF. We outline Gaposhkin's proof for the sake of completeness. Let us suppose that $n_{1}<\cdots<n_{k-1}$ and $f_{1}, \ldots, f_{k-1}$ have been defined so that $\left\|h_{j}-f_{j}\right\|_{p}<$ $2^{-j}$, and if $j \geq 2, E\left(f_{j}, \Sigma_{j-1}\right)=0$, where $\Sigma_{j}$ is the algebra generated by the simple functions $f_{1}, \ldots, f_{j}$, and $h_{j}=g_{n_{j}}$, for $j=1, \ldots, k-1$. As $\lim _{n}\left\|E\left(g_{n}, \Sigma_{k-1}\right)\right\|_{p}=$ 0 , we can choose $n_{k}>n_{k-1}$ with $\left\|E\left(h_{k}, \Sigma_{k-1}\right)\right\|_{p}<2^{-k-2}$, where $h_{k}=g_{n_{k}}$. We take a simple function $f_{k}^{\prime}$ so that $\left\|f_{k}^{\prime}-h_{k}\right\|_{p}<2^{-k-2}$. Then by setting $f_{k}=f_{k}^{\prime}-E\left(f_{k}^{\prime}, \Sigma_{k-1}\right)$ we can go on with the induction.

Suppose now that we knew that $\left(f_{n}\right)$ were Cesàro converging to zero in the $L^{p}$ norm. Then as the series in Theorem A is converging, we would easily obtain from the following inequality that $\left(h_{n}\right)$ was also Cesàro converging to zero:

$$
\left\|n^{-1} \sum_{k=1}^{n} h_{k}\right\|_{p} \leq\left\|n^{-1} \sum_{k=1}^{n}\left(h_{k}-f_{k}\right)\right\|_{p}+\left\|n^{-1} \sum_{k=1}^{n} f_{k}\right\|_{p} .
$$

Received by the editors December 24, 1986 and, in revised form, March 9, 1987.

1980 Mathematics Subject Classification (1985 Revision). Primary 46E30, 60F25; Secondary 28A20.

Key words and phrases. Martingale difference sequence, Cesàro convergence in the $L^{p}$ norm, weakly null sequences in $L^{p}$. 
Thus we get the theorems proved by Banach and Saks in [BS] and by Szlenk in $[\mathbf{S}]$, asserting that any weakly null sequence in $L^{p}$ admits a subsequence which is Cesàro converging in the norm, from this remark and the following

THEOREM. If $\left(f_{n}\right)$ is an m.d.s. in $L^{p}$ which is weakly null in $L^{p}$, then its arithmetic means converge to zero in the $L^{p}$ norm.

PROOF. The proof in the case of $p>1$ is essentially contained in Banach and Saks' paper [BS], and it is based on the inequality

$$
|s+t|^{p} \leq|s|^{p}+p|s|^{p-1} \operatorname{sgn}(s) t+\sum_{k=2}^{[p]}\left(\begin{array}{l}
p \\
k
\end{array}\right)|s|^{p-k}|t|^{k}+A|t|^{p}
$$

which holds for any real numbers $s$ and $t$, and in which $A$ is a finite constant and $[p]$ is the greatest integer less than or equal to $p$.

Using this inequality and assuming that $\left(f_{n}\right)$ is an m.d.s. adapted to $\left(\Sigma_{n}\right)$ with $\left\|f_{n}\right\|_{p} \leq 1$, we obtain $\left\|S_{n}\right\|_{p} \leq\left\|S_{n-1}\right\|_{p}+B n^{p-2}+A$, where $S_{n}=\sum_{k=1}^{n} f_{k}$ and $B$ is a finite constant, hence obtaining $\lim \left\|n^{-1} S_{n}\right\|_{p}=0$, as shown by Banach and Saks.

We notice that

$$
p \int\left|S_{n-1}(x)\right|^{p-1} \operatorname{sgn}\left(S_{n-1}(x)\right) f_{n}(x) d x
$$

vanishes because $\left|S_{n-1}\right|^{p-1} \operatorname{sgn}\left(S_{n-1}\right)$ is $\Sigma_{n-1}$-measurable and is in $L^{q}$, if $p^{-1}+$ $q^{-1}=1$.

Now we assume that $p=1$ and that $\left(f_{n}\right)$ is an equi-integrable m.d.s. in $L^{1}$ adapted to $\left(\Sigma_{n}\right)$ with $\left\|f_{n}\right\|_{1} \leq 1$ for every $n$.

Let us fix $\varepsilon>0$, and let $M$ be a finite constant to be fixed later. Let $g_{n}=$ $f_{n} \chi\left(\left|f_{n}\right| \leq M\right)$, where, as usual, $\chi_{\left(\left|f_{n}\right| \leq M\right)}$ is the characteristic function of the set $\left(\left|f_{n}\right| \leq \bar{M}\right)=\left\{x:\left|f_{n}(x)\right| \leq M\right\}$.

Let $h_{n}=E\left(g_{n}, \Sigma_{n-1}\right)$ for $n>1$, and $h_{1}=g_{1}$. Then $h_{n}$ is $\Sigma_{n-1}$-measurable, $\left(g_{n}-h_{n}\right)$ is an m.d.s. adapted to $\left(\Sigma_{n}\right)$ and $\left\|g_{n}-h_{n}\right\|_{\infty} \leq 2 M$.

Let $S_{n}=\sum_{k=1}^{n} f_{k}, S_{n}^{\prime}=\sum_{k=1}^{n}\left(f_{k}-g_{k}\right), S_{n}^{\prime \prime}=\sum_{k=1}^{n}\left(g_{k}-h_{k}\right)$, and $S_{n}^{\prime \prime \prime}=$ $\sum_{k=1}^{n} h_{k}$.

Then

$$
\begin{aligned}
\left\|S_{n}\right\|_{1} & \leq\left\|S_{n}^{\prime}\right\|_{1}+\left\|S_{n}^{\prime \prime}\right\|_{1}+\left\|S_{n}^{\prime \prime \prime}\right\|_{1} \\
& \leq \sum_{k=1}^{n}\left\|f_{k} \chi_{\left(\left|f_{k}\right|>M\right)}\right\|_{1}+\left\|S_{n}^{\prime \prime}\right\|_{2}+\sum_{k=1}^{n}\left\|h_{k}\right\|_{1} .
\end{aligned}
$$
$L^{2}$.

We have $\left\|S_{n}^{\prime \prime}\right\|_{2}^{2}=\sum_{k=1}^{n}\left\|g_{k}-h_{k}\right\|_{2}^{2} \leq 4 n M^{2}$, because $\left(g_{n}-h_{n}\right)$ is an m.d.s. in

As $\left(f_{n}\right)$ is equi-integrable, we can choose $\delta>0$ such that for any measurable $A$ whose measure does not exceed $\delta, \int_{A}\left|f_{n}(x)\right| d x \leq \varepsilon / 3$ for every $n$. Then, we fix $M$ with $M>\delta^{-1}$, obtaining $\left\|f_{n} \chi\left(\left|f_{n}\right|>M\right)\right\|_{1} \leq \varepsilon / 3$ for every $n$. 
Furthermore, let $u$ be a $\Sigma_{n-1}$-measurable and essentially bounded function. Then,

$$
\begin{aligned}
\int h_{n}(x) u(x) d x & =\int f_{n}(x) u(x) \chi_{\left(\left|f_{n}\right| \leq M\right)}(x) d x \\
& =-\int f_{n}(x) u(x) \chi_{\left(\left|f_{n}\right|>M\right)}(x) d x .
\end{aligned}
$$

Thus $\left|\int h_{n}(x) u(x) d x\right| \leq\|u\|_{\infty}\left\|f_{n} \chi_{\left(\left|f_{n}\right|>M\right)}\right\|_{1}$, and so $\left\|h_{n}\right\|_{1} \leq \varepsilon / 3$, since $h_{n}$ is $\Sigma_{n-1}$-measurable.

Putting all the parts together, we obtain

$$
\left\|S_{n}\right\|_{1} \leq n \varepsilon / 3+2 M n^{1 / 2}+n \varepsilon / 3
$$

finishing the proof.

\section{REFERENCES}

[BS] S. Banach and S. Saks, Sur la convergence forte dans les champs $L^{p}$, Studia Math. 2 (1930), 51-57.

[G] V. F. Gaposhkin, Convergence and limits theorems for sequences of random variables, Theor. Probab. Appl. 17 (1972), 379-399.

[S] W. Szlenk, Sur les suites faiblement convergentes dans l'espace L, Studia Math. 25 (1965), 337-341.

Facultad de Matemáticas, Universidad de Sevilla, 41012-Sevilla, España 